\title{
Stephan Klecha
}

\section{Zum Zusammenhang von Wahlrechtsreformen und Parteiensystem}

\author{
Eine Debatte um Henne und Ei
}

Wahlsystemdebatten sind in der Bundesrepublik selten geworden und die Zufriedenheit mit dem vorhandenen Wahlsystem wird mittlerweile als recht hoch eingeschätzt. ${ }^{1}$ Das war nicht immer so. Vor allem während der 1950er und 1960er Jahre stand die Grundsatzfrage im Raum, ob das Verhältniswahlrecht der Weimarer Republik das Parteiensystem zersplittert und darüber den Aufstieg radikaler Flügelparteien begünstigt habe. ${ }^{2}$ Dieses bejahend war die Forderung nach einem mehrheitsbildenden Wahlsystem die logische Folge für die sich konsolidierende Bundesrepublik. Mit dem Scheitern eines derartigen Reformvorhabens am Ende der 1960er Jahre war das Thema danach lange Zeit von der politischen Tagesordnung verschwunden. Die vorgebrachten Argumente waren in Anbetracht der Stabilität und Konzentration des Parteiensystems sowieso Makulatur geworden. ${ }^{3}$

In den letzten 20 bis 30 Jahren hat es nun eine Reihe von Veränderungen im Parteiensystem gegeben, die die Mehrheitsbildung tangieren. Obwohl damit die einstigen normativen Argumente wieder relevant werden, ist eine grundlegende Wahlsystemdebatte ausgeblieben. ${ }^{4}$

Dabei liegt der Gedanke nahe, den Zusammenhang von Parteien- und Wahlsystem nochmals zu überprüfen. Es mag erst einmal eine triviale Erkenntnis sein, dass beide Systeme in einem gewissen Abhängigkeitsverhältnis zueinander stehen. Parteien, die sich erfolgreich in Wahlen behaupten wollen, müssen die Besonderheiten des Wahlsystems

1 Eckhard Jesse, »Verhältniswahl und Gerechtigkeit « in: Gerd Strohmeier (Hg.), Wablsystemreform, Baden-Baden 2009, S. 105-131, hier S. 127; Manfred Schmidt, Das politische System Deutschlands, Institutionen, Willensbildung und Politikfelder, Bonn 2010, S. 51.

2 Dolf Sternberger, Die große Wablreform, Zeugnisse einer Bemübung, Köln u.a. 1964; Ferdinand A. Hermens, Demokratie oder Anarchie, Untersuchung über die Verhältniswabl, Köln/Opladen 1968, 2. Auflage; Siehe auch Maximilian Steinbeis, / Marion Detjen, / Stephan Detjen, Die Deutschen und das Grundgesetz, Geschichte und Grenzen unserer Verfassung, Bonn 2009, 139.

3 Wilhelm Hennis, Regieren im modernen Staat, Politikwissenschaftliche Abbandlungen I, Tübingen 1999, S. 248; Frank Decker, »Die Bundesrepublik auf der Suche nach neuen Koalitionen « in: Aus Politik und Zeitgeschichte, 35-36/2007, S. 26-33, hier S. 26; Roman Herzog, Strukturmängel der Verfassung? Erfabrungen mit dem Grundgesetz, Stuttgart/München, S. 43; Volker von Prittwitz, »Vollständig personalisierte Verhältniswahl « in: Aus Politik und Zeitgeschichte, 52/2007, S. 12-20, hier S. 14.

4 Joachim Behnke, Das Wahlsystem der Bundesrepublik Deutschland, Logik, Technik und Praxis der Verbältniswabl, Baden-Baden 2007, 13. 
antizipieren. Das Wahlsystem könnte deswegen die unabhängige Variable sein, welche das Parteiensystem beeinflusst. ${ }^{5}$ Im Anschluss an Maurice Duverger ${ }^{6}$ wird sehr generalisierend festgehalten, dass Mehrheitswahlsysteme tendenziell eher mit einem stärker konzentrierten Parteiensystem einhergehen, wohingegen ein Verhältniswahlsystem eher ein fragmentiertes Parteiensystem erwarten lässt. Eine zunehmende Fragmentierung des Parteiensystems würde vice versa die Verhältniswahl weiter stabilisieren, weil das Wahlsystem aus dem existierenden Parteiensystem heraus entsteht. Selbst diese scheinbar naheliegenden Feststellungen lassen sich in einer vergleichenden Perspektive schwerlich halten. ${ }^{7}$

Dreht man das Argument um und hält das Parteiensystem für die unabhängige Variable, die zur Herausbildung eines spezifischen Wahlsystems führt, ${ }^{8}$ so kann man hierfür ebenso gute Anknüpfungspunkte finden. Die im Parlament vertretenden Parteien sind schließlich in der Lage, das Wahlsystem für sich selbst möglichst günstig zu gestalten. Es handelt sich also um eine Wettbewerbsordnung eines herrschenden Oligopols. In der Konsequenz verstetigt das Wahlsystem deswegen idealerweise ein vorgefundenes Parteiensystem, was aber in der Regel nie ganz gelingt, weil neue Parteien hinzutreten können.

Wie man es dreht und wendet, es gibt genügend Anhaltspunkte und Belege sowohl für die These, wonach das Parteien- das Wahlsystem beeinflusst, als auch für den Gedanken, dass das Parteiensystem durch das Wahlsystem vorstrukturiert wird. Der folgende Beitrag will dieser immergrünen Debatte neues Material beisteuern, indem die Wandlungen des Parteiensystems der letzten zwanzig Jahre auf Bundesebene mit den Entwicklungen im Wahlsystem abgeglichen werden. Während dabei die Forderung nach einem grundlegenden Wechsel im Wahlsystem ${ }^{9}$ eher randständig ist, wird die Debatte in erster Linie von Funktionsdefekten geprägt, ${ }^{10}$ die erst mit dem Aufbrechen des Parteiensystems virulent geworden sind.

Im Zentrum des Interesses steht dabei die an Duverger anknüpfende normative Vorstellung, wonach über eine Veränderung des Wahlsystems das Parteiensystem so geformt werden kann, dass die parlamentarische Mehrheitsbildung erleichtert wird. Diese Her-

5 Giovani Sartori, »The Party-Effects of Electoral Systems «, in: Hazan, Reuven Y. / Maor, Moshe (Hg.), Parties, Elections and Cleavages: Israel in Comparative and Theoretical Perspective, London/Portland 2000, S. 13-28, S. 13ff., ders., Comparative Constitutional Engineering. An Inquiry into Structures, Incentives and Outcome, New York 1994.

6 Maurice Duverger, Die politischen Parteien, Tübingen 1959, Hermens, Demokratie oder Anarchie, Untersuchung über die Verbältniswabl, aaO. (FN 2).

7 Dieter Nohlen, Wablrecht und Parteiensystem, Zur Theorie und Empirie der Wablsysteme, Opladen/Farmington Hills 2009; Klaus Poier, »Wahlsysteme im internationalen Vergleich ein Überblick « in: ders. (Hg.), Demokratie im Umbruch, Perspektiven einer Wablrechtsreform, Wien/Köln/Graz 2009, S. 41-73; Foroud Shirvani, Das Parteienrecht und der Strukturwandel im Parteiensystem, Tübingen 2010, S. 415; Tim Baedermann, Der Einfluss des Wablrechts auf das Parteiensystem, Baden-Baden 2007.

8 Seymoure Lipset, / Stein Rokkan (Hg.), Party Systems and Voter Alignments, New York 1967.

9 Jesse, »Verhältniswahl und Gerechtigkeit«aaO. (FN 1), S. 114.

10 Frank Decker, Regieren im »Parteienbundesstaat". Zur Architektur der deutschen Politik, Wiesbaden 2011, S. $145 \mathrm{ff}$. 
angehensweise fokussiert nur auf eine Funktion des Wahlsystems, ${ }^{11}$ doch es scheint fruchtbar zu sein, sich damit näher zu befassen, nachdem das Bundesverfassungsgericht eine Reform des Wahlrechts angemahnt hat. Wahlrecht und Wahlsystem werden oftmals synonym verwendet, wiewohl zwischen beidem zu differenzieren ist. ${ }^{12}$ Das Wahlsystem beschreibt einen wesentlich kleineren Ausschnitt als das Wahlrecht. ${ }^{13}$ Während das Wahlrecht die Gesamtheit aller Angelegenheiten beschreibt, die zur Organisation der Wahl notwendig sind, befasst sich das Wahlsystem allgemein mit der Frage, inwieweit die Stimmen der Wähler in politische Repräsentation übersetzt werden. ${ }^{14}$ Eine Debatte um eine Reform des Wahlrechts kann, muss aber nicht zwingend, auch Elemente einer Wahlsystemdiskussion beinhalten. ${ }^{15}$

Vor diesem Hintergrund müsste sich in der laufenden Debatte auch die Triebfeder der Entwicklung finden lassen. Leiten sich die nun in Rede stehenden Veränderungen des Wahlrechts eher aus dem Wandlungsprozess im Parteiensystem ab oder ist es genau umgekehrt? Dazu werden drei zentrale Wegmarken aufgegriffen, nämlich die Bundestagswahlen 1994, 1998 und 2005, ehe dann auf die aktuelle Situation Bezug genommen wird.

\section{Die Bundestagswabl 1994: Rettung der PDS und Streit um Überhangmandate}

Das PDS-Wählerpotential in Ostdeutschland hätte für die SED-Nachfolgerin kaum ausgereicht, um bei den ersten gesamtdeutschen Bundestagswahlen die Sperrklausel zu überwinden. CDU/CSU/FDP-Regierungskoalition und SPD-Opposition hofften daher, die PDS frühzeitig als bundespolitische Kraft auszuschalten. ${ }^{16}$ Das Wahlsystem sollte also an dieser Stelle ganz eindeutig das Parteiensystem prägen, in dem es der PDS wahrscheinlich die Parlamentswürdigkeit abgesprochen hätte.

Bei der Bundestagswahl 1990 fand aber einmalig wegen eines Urteils des Bundesverfassungsgerichts die Sperrklausel in Ost- und Westdeutschland getrennt Anwendung. Die PDS konnte damit für zunächst eine Legislaturperiode ihre bundespolitische Exis-

11 Nohlen, Wablrecht und Parteiensystem, Zur Theorie und Empirie der Wablsysteme, aaO. (FN 7), S. $169 \mathrm{ff}$.

12 Decker, Regieren im »Parteienbundesstaat «. Zur Architektur der deutschen Politik, aaO. (FN 10), S. $135 \mathrm{ff}$.

13 Nohlen, Wablrecht und Parteiensystem, Zur Theorie und Empirie der Wablsysteme, aaO. (FN 7), S. 61.

14 Matthias Catón, Wablsysteme und Parteiensystem im Kontext, Vergleichende Analyse der Wirkung von Wablsystemen unter verschiedenen Kontextbedingungen, Heidelberg 2009, <http:// www.caton.de/publikationen/buecher/wahlsysteme-und-parteiensysteme.html> (Stand: 17.5.2011), S. 31 .

15 Gerd Strohmeier, »Vergangene und zukünftige Reformen des deutschen Wahlsystems « in: ders. (Hg.), Wablsystemreform, Baden-Baden 2009, S. 11-44; Franz Urban Pappi / Michael Herrmann, »Überhangmandate ohne negatives Stimmgewicht: Machbarkeit, Wirkungen, Beurteilungen « in: Zeitschrift für Parlamentsfragen, 2/2009, S. 312-322; Florian Grotz, »Verhältniswahl und Regierbarkeit « in: Gerd Strohmeier (Hg.), Wablsystemreform, Baden-Baden, S. 155-181; v. Prittwitz, »Vollständige personalisierte Verhältniswahl«, aaO. (FN 3), S. 12-20.

16 Eckhard Jesse, Demokratie in Deutschland. Diagnosen und Analysen, Köln/Weimar/Wien 2008. 
tenz sichern. Selbiges galt knapp für Bündnis 90 , dessen Fusion mit den westdeutschen Grünen in Anbetracht der 1994 gesamtdeutsch wirkenden Sperrklausel beschleunigt wurde. Die Bedingungen des Wahlrechts begünstigten grundsätzlich eine gesamtdeutsche Struktur von Parteien, was bei der PDS an einem hinreichend potenten Partner im Westen scheiterte. Zwar hatte sie sich als Stimme Ostdeutschlands zu profilieren versucht, doch das vermeintliche Ost-West-Cleavage reichte kaum aus, um aus eigener Kraft die $5 \%$-Hürde zu überspringen.

Allerdings hatte der starke Ostbias die Partei derart stabilisiert, dass sie in einigen Wahlkreisen, Chancen auf die Erlangung eines Direktmandates hatte. Nun sieht das Bundeswahlgesetz die Regelung vor, dass die Fünfprozenthürde umgangen werden kann, wenn eine Partei mindestens drei solcher Mandate erringt. Diese Regelung war ursprünglich eher zufällig ins Wahlrecht gelangt, wurde aber beibehalten, um der Deutschen Partei als Koalitionspartner der Union auf Bundesebene in den 1950er Jahren das parlamentarische Überleben zu sichern. ${ }^{17}$

Nun hatte die PDS aber keinen machtpolitischen Nutzen für die Mehrheitsparteien im Bundestag. Vielmehr bedrohte eine starke PDS die Wiederwahlchancen der amtierenden christ-liberalen Koalition (und minderte auch die Chancen des rot-grünen Oppositionslagers auf einen Regierungswechsel). Es wäre aus Sicht der etablierten Parteien demnach naheliegend gewesen, das Wahlrecht an dieser Stelle anzupassen und so die Stimmen für die PDS bei der Mandatszuteilung wahrscheinlich zu eliminieren. Doch zu einem solchen Schritt konnten sich die Regierungsparteien unmöglich durchringen.

Die Wiedervereinigung hatte nämlich das gewöhnliche, bundesweite Stimmengewicht der CSU auf rund sieben Prozent gemindert. Schlechte Umfragewerte rückten die Partei 1993/94 in die Nähe zur Fünfprozenthürde. ${ }^{18}$ Mithin war der Erhalt der Direktmandatsklausel für die CSU zwingend. Deswegen blieb das Bundestagswahlrecht insoweit unverändert und die PDS konnte durch den Gewinn von vier Direktmandaten erneut in den Deutschen Bundestag einziehen.

Es lässt sich damit für diese Wahl eine wechselseitige Beeinflussung von Wahl- und Parteiensystem feststellen. Wie zuvor schon die Grünen, musste die PDS die Vorgaben des Wahlsystems antizipieren, um ihre spezifische Stärke zu maximieren. Gleichzeitig war das Parteiensystem nicht in der Lage, das Wahlsystem anzupassen, um die PDS auszuschalten.

Bei der Wahl fielen zudem bei der CDU in beträchtlichem Umfang Überhangmandaten an. Überhangmandate entstehen dem Grunde nach, wenn eine Partei mehr Direktmandate gewinnt, als ihr rechnerisch nach dem Z weitstimmenanteil zustehen. Dieser Effekt tritt jedoch - bei einer bisherigen Ausnahme - nur als Resultat der Verrechnung des bundesweiten Wahlergebnisses auf die Landeslisten der Parteien auf. ${ }^{19}$ Bei diesen internen Überhangmandaten handelt sich letztlich um einen Fehler im Verrechnungs-

17 Jesse, »Verhältniswahl und Gerechtigkeit«aaO. (FN 1), S. 123.

18 Andreas Kießling, Die CSU, Machterbalt und Machterneuerung, Wiesbaden 2004, S. 257.

19 Behnke, Das Wablsystem der Bundesrepublik Deutschland. Logik, Technik und Praxis der Verbältniswabl, aaO. (FN 4), S. 183. 
verfahren. Das wird daran deutlich, dass ja weiterhin so viele Direktmandate wie Wahlkreise zugeteilt werden, dafür aber mehr Listenmandate entstehen. Behnke spricht in diesem Zusammenhang vom »wahlsystematischen Wurmloch $\ll{ }^{20}$ Da sich Überhangmandate insofern auch nicht als Prämie besonders erfolgreicher Landesverbände interpretieren lassen, ${ }^{21}$ ist nicht ersichtlich, dass sie systematisch im Wahlsystem angelegt wurden.

1994 verstärkte diese Anomalie dank des rationalen Stimmensplittings der Wähler beider Parteien ${ }^{22}$ nun die sehr knappe Mandatsmehrheit von Union und FDP. Der nunmehr offenbar mehrheitsbeeinflussende Charakter der Überhangmandate veranlasste die SPD-Opposition zu einer Klage vor dem Bundesverfassungsgericht, um die Vereinbarkeit der Überhangmandate mit dem Grundgesetz zu überprüfen. Die Richter wiesen den Klageantrag zunächst $a b,{ }^{23}$ befanden später aber, dass es für ausscheidende Mandatsträger eines davon betroffenen Landesverbands keine Nachbesetzung geben dürfe, solange der proportionale Mandatsanteil nicht hergestellt ist.

\section{Gewöhnung an Überhangmandate und das zwischenzeitliche Aus für die PDS: 1998 bis 2005}

Auf dieser Grundlage hatten 13 Überhangmandate der SPD bei der Bundestagswahl 1998 auch die neue rot-grüne Mehrheit beträchtlich anwachsen lassen. Nachdem also die neue Koalition von einer Regelung profitieren durfte, die sie einst in Frage gestellt hatte, versteht es sich fast von selbst, dass der Reformbedarf für die neue Mehrheit gedeckt war. Zumal der entsprechende Handlungsdruck auch so nicht allzu groß war, weil eine Verkleinerung des Bundestags nebst Neuzuschnitt der Wahlkreise mit Hinblick auf die Wahl 2002 bereits beschlossen war. Weniger Überhangmandate waren dabei wahrscheinlich, weil die Wahlkreisabgrenzung als Hauptursache der Überhangmandate der Jahre 1990-1998 angesehen wurde. ${ }^{24}$

In der Tat reduzierte sich die Zahl der Überhänge 2002 erwartungsgemäß. Erkennbar wurde wegen des knappen Wahlergebnisses aber die Gefahr einer möglichen Umkehrung der Mehrheitsverhältnisse durch Überhangmandate. ${ }^{25}$ Zudem hing die verminderte Zahl der Überhangmandate vor allem damit zusammen, dass die PDS die Sperrklausel gerissen

20 Ebd., S. 184; außerdem Heinrich Pehle, »Die Bundestagswahlen von 2005 und 2009: Verfassungswidrig und doch gültig «, in: Gesellschaft - Wirtschaft - Politik, 4/2008, S. 471-478.

21 Joachim Behnke, »Überhangmandate: Ein (behebbarer) Makel im institutionellen Design des Wahlsystems « in: Zeitschrift für Politikwissenschaft, 3/2003, S. 1235-1269, hier: S. 1253.

22 Joachim Behnke, »Überhangmandate bei der Bundestagswahl 2009. Eine Schätzung mit Simulationen « in: Zeitschrift für Parlamentsfragen, 3/2009, S. 620-636.

23 Jesse, Demokratie in Deutschland. Diagnosen und Analysen, aaO. (FN 16), S. 232; Behnke, »Überhangmandate bei der Bundestagswahl 2009. Eine Schätzung mit Simulationen« aaO. (FN 22), S. 621.

24 Behnke, Das Wablsystem der Bundesrepublik Deutschland, Logik, Technik und Praxis der Verbältniswahl, aaO. (FN 4), S. 189.

25 Ebd., S. 42. 
hatte und aus der Mandatszuteilung ausgeschieden war. ${ }^{26}$ Das wiederum hatte tatsächlich seine Ursache im Neuzuschnitt der Wahlkreise, der noch zu Zeiten der Regierung Kohl erfolgte. Dabei waren nämlich die von der PDS gewonnenen Wahlkreise so neu abgegrenzt worden, dass das Schlupfloch im Wahlsystem für die Partei enger wurde. Fortan waren zwei Wahlkreise für die Partei absolut sicher, weitere waren aber bei einem durchschnittlichen Stimmenergebnis aussichtslos. Deswegen konnte die PDS dann 2002 auch nur noch diese zwei Mandate gewinnen und verpasste den Einzug in den Bundestag. Veränderungen scheinbar minderer Ordnung im Wahlsystem implizieren also bestimmte Effekte im Parteiensystem. Der Wegfall der PDS 2002 spricht also dafür, dass das Wahldas Parteiensystem ausformt.

Der weitere Verlauf spricht dafür, dass das Parteiensystem sich weiter an das Wahlsystem anpasste. Die PDS ging bei der vorgezogenen Bundestagswahl 2005 rasch eine faktische Listenverbindung mit der SPD-Abspaltung WASG ein, weil ein gleichzeitiger Antritt beider Formationen sehr wahrscheinlich zu einem Scheitern beider geführt hätte. ${ }^{27}$ Das Wahlsystem katalysierte also die Bildung einer neuen Linkspartei und schuf dadurch eine Gelegenheitsstruktur, um die zuvor nicht erfolgreiche Westausdehnung der Partei nachzuholen.

Vor diesem Hintergrund gestaltet die Bundestagswahl 2005 die Parteienlandschaft nachhaltig um, was nicht ohne Folgen für das Wahlsystem selbst blieb. Union und SPD verloren bei dieser Wahl gleichermaßen Stimmen. Zugleich gelang der um die WASG angereicherten Linkspartei.PDS sowohl bei den Zweitstimmen als auch durch drei Direktmandate der Sprung über die Sperrklausel. Das Wiederhinzutreten der PDS bei der Mandatszuteilung ließ die Zahl der Übergangmandate wieder beträchtlich anwachsen.

Neun Überhangmandate für die SPD und sieben für die CDU hätten am Ende sogar fast dazu geführt, dass die SPD trotz geringeren Zweitstimmenanteils mehr Mandate als die Unionsparteien besessen hätte. ${ }^{28}$ Während seit 1987 die Überhangmandate faktisch wie eine Art Mehrheitsprämie für die stimmenstärkste Partei (unter der Annahme, CDU und CSU an dieser Stelle als eine Partei zu betrachten) wirkten, war dies 2005 widerlegt worden. ${ }^{29}$ Eine Umkehrung der in der Verhältniswahl mit der Zweitstimme ermittelten eigentlichen Mehrheitsverhältnisse war somit immer wahrscheinlicher geworden. Außerdem kompensierten die beiden altbundesrepublikanischen Lager Rot-Grün und Schwarz-Gelb dadurch ein wenig ihre Schwäche in Ostdeutschland. Es gibt also ganz deutliche Anzeichen dafür, dass das Wahlsystem bis hierher maßgeblich das Parteiensystem der wiedervereinigten Bundesrepublik beeinflusst hat.

26 Joachim Behnke, »Ein integrales Modell der Ursachen von Überhangmandaten« in: Politische Vierteljabresschrift, 1/2003, S. 41-65, hier: S. 62.

27 Gero Neugebauer / Richard Stöss, »Die Partei Die Linke. Nach der Fründung in des Kaisers neuen Kleidern? Eine politische Bedarfsgemeinschaft als neue Partei im deutschen Parteiensystem « in: Oskar Niedermayer (Hg.), Die Parteien nach der Bundestagswahl 2005, Wiesbaden 2008, S. 151-200, hier: S. 154ff.

28 Jesse, »Verhältniswahl und Gerechtigkeit«aaO. (FN 1), S. 126.

29 Grotz, »Verhältniswahl und Regierbarkeit«aaO. (FN 15), S. 163.

ZfP 58. Jg. 3/2011 
Allerdings hatte das Wahlsystem es eben nicht geschafft, das Fünfparteiensystem zu verhindern. Aus diesem Grund wurden einige bis dahin belanglose Funktionsdefekte offenkundig, die für die demokratische Legitimation der Wahl als problematisch einzustufen sind. ${ }^{30}$ Deswegen spricht einiges dafür, dass nunmehr das Parteiensystem auf das Wahlsystem wirkt. Das Wahlsystem bildet jedenfalls nicht mehr adäquat die Struktur des Parteiensystems ab.

\section{Aporien des Wablrechts: Negatives Stimmengewicht 2005}

Nach der Bundestagswahl 2005 hat es deswegen wieder eine ernste Debatte in der Politik um das Wahlrecht und das Wahlsystem gegeben. Aufgegriffen wurden zunächst einmal geringfügige Änderungen im Zuteilungsverfahren, die verhindern, dass eine Partei bei einer Veränderung der Stimmenverteilung entgegen des zu erwartenden Effekts mehr oder weniger Mandate erhält. ${ }^{31}$ Damit wurde aber ein Problem nicht beseitigt, dass den Ursprung der gegenwärtigen Debatte ausmacht, das negative Stimmengewicht.

In einem Dresdener Wahlkreis fand zeitversetzt und im Angesicht des vorliegenden vorläufigen Endergebnisses eine Nachwahl statt, bei welcher der dortige CDU-Bewerber nur über ein gewonnenes Direktmandat in den Bundestag einziehen konnte, weil die CDU in Sachsen bereits Überhangmandate erzielt hatte. Der Gewinn des Direktmandats war als sicher einzustufen und hätte somit eigentlich ein weiteres Überhangmandat zur Folge. Allerdings hätte die gleichzeitig zu erwartende Zweitstimmenzahl der CDU paradoxerweise einen Mandatsverlust eingebracht. Hätte die CDU nämlich die realistische Größenordnung von rund $42.000 \mathrm{Zweitstimmen} \mathrm{oder} \mathrm{mehr} \mathrm{erlangt,} \mathrm{so} \mathrm{wäre} \mathrm{das} \mathrm{hinzu-}$ gewonnene Direktmandat durch ein weiteres Listenmandat für die CDU in Sachsen kompensiert worden. Allerdings handelte es sich hierbei nicht um ein Nullsummenspiel.

Das Wahlrecht zum Deutschen Bundestag unterscheidet nämlich zwischen einer Oberverteilung, welche die Verteilung der Mandate zwischen den Parteien festlegt, und einer Unterverteilung, bei der die in der Oberverteilung ermittelten Mandate einer Partei auf deren Landeslisten verteilt werden. Durch die Koppelung von Ober- und Unterverteilung wäre der CDU-Mandatszugewinn in Sachsen nun nicht bundesweit entstanden, sondern alleine durch die veränderte Relation zwischen den CDU-Landeslisten. Das bedeutet, das zusätzliche Mandat der CDU in Sachsen wäre allein durch den Wegfall eines CDU-Mandats in einem anderen Land (Nordrhein-Westfalen) hinzugewonnen worden. Im Ergebnis hätte die CDU also für den Zugewinn eines Direktmandats nicht

30 Behnke, »Ein integrales Modell der Ursachen von Überhangmandaten« aaO. (FN 25), S. 64; Hans Meyer, Die Zukunft des Bundestagswablrechts. Zwischen Unverstand, obiter dicta, Interessenkalkül und Verfassungsverstoß, Baden-Baden 2010, S. 54.

31 Behnke, »Überhangmandate: Ein (behebbarer) Makel im institutionellen Design des Wahlsystems « aaO. (FN 21), S. 1242; Friedrich Pukelsheim, »Bundeswahlgesetz - nächste Etappe in: Deutsches Verwaltungsblatt, 14/2008, S. 889-897; Friedrich Pukelsheim, »Erfolgswertgleichheit der Wählerstimmen zwischen Anspruch und Wirklichkeit« in: Die öffentliche Verwaltung, 10/2004, S. 405-414; Pehle, »Die Bundestagswahlen von 2005 und 2009: Verfassungswidrig und doch gültig? «aaO. (FN. 20). 
nur eine Kompensation auf der Landesliste erfahren, sondern zugleich ein Mandat in Nordrhein-Westfalen verloren. Eine Zweitstimme für die CDU bei dieser Nachwahl hätte also der Partei nicht genutzt, sondern objektiv geschadet. In Kenntnis dessen reagierten die Wähler mit ihrem Votum ganz offensichtlich auf dieses Phänomen und verhinderten durch faktische Stimmenthaltung bei der Zweitstimme den Mandatsverlust für die CDU. ${ }^{32}$

Obwohl dieser Effekt auch bei früheren Wahlen auftrat, ignorierten ihn das Verfassungsgericht und auch die meisten Wahlrechtsexperten weitgehend. ${ }^{33}$ Tatsächlich wussten die Wähler zum Zeitpunkt der Stimmabgabe schlicht nicht, ob die Stimme für eine Partei ihr möglicherweise am Ende schaden könnte. Zunächst einmal nutzt ja auf der Ebene der Oberverteilung eine Stimme stets der gewählten Partei. Erst aus dem Zusammenspiel von Ober- und Unterverteilung in Verbindung mit dem Entstehen von Überhängen kann das negative Stimmengewicht schließlich Wirkung entfalten und es ist überdies nur auf eine sehr geringe Zahl von Mandaten begrenzt.

$\mathrm{Da}$ in der Regel nicht bekannt war, ob und wo Überhangmandate auftreten, konnte auch weder auf deren Entstehen spekuliert noch abgeschätzt werden, ob daraus am Ende sogar ein negatives Stimmengewicht resultieren könnte. Bei der Bundestagswahl 2005 hatte sich das verändert. In Internetforen wurden bereits im Vorfeld der Hauptwahl ausgiebig Empfehlungen gegeben, wann und wo Überhangmandate auftreten konnten. Die Wähler konnten dabei nicht nur in Hinblick auf diese Möglichkeit der Mandatsmaximierung eines Lagers votieren, sondern sogar abschätzen, ob sie mit ihrer Stimme nicht bewusst gegen ihre eigene Parteipräferenz abstimmen sollten, um ihrer Partei damit zu nutzen. In besonders deutlicher Form war dies dann in Dresden möglich. Es ist Zufall, dass die dortige Nachwahl mit dem endgültigen Aufbrechen des Parteiensystems zusammengefallen ist. Tatsächlich hat der geringere Wählerzuspruch für Union und SPD im Fünfparteiensystem die Absurdität des deutschen Wahlsystems erst offenbart und relevant werden lassen. Kurzum, das Parteiensystem passte nicht mehr vollständig zum Wahlsystem.

Eine Wahlprüfungsbeschwerde vor dem Bundesverfassungsgericht in dieser Sache hatte schließlich Erfolg. Es sei nach Auffassung des Gerichts nicht hinzunehmen, dass eine Stimme für eine Partei dieser im Ergebnis schaden könne. Die Richter revidierten damit teilweise ihre einstige Entscheidung aus dem Jahr 1997. ${ }^{34}$ Die notwendige, wenn auch nicht hinreichende Bedingung, wonach interne Überhangmandate Ursache des negativen Stimmengewichts sind, wurde dennoch nicht angetastet.

Durch die vom Verfassungsgericht gegebene lange Frist für eine Wahlrechtsnovelle war der Raum für das politisch-taktische Kalkül der Parteien geöffnet. Eine Regelung

32 Strohmeier, »Vergangene und zukünftige Reformen des deutschen Wahlsystems«aaO. (FN 15), S. 31.

33 Pehle, »Die Bundestagswahlen von 2005 und 2009: Verfassungswidrig und doch gültig «aaO. (FN 20), S. $473 \mathrm{f}$.

34 BVerfGE 121, 266; Strohmeier, »Vergangene und zukünftige Reformen des deutschen Wahlsystems « aaO. (FN 15), S. 28, 34; Pehle, »Die Bundestagswahlen von 2005 und 2009: Verfassungswidrig und doch gültig", aaO. (FN 20), S. 476. 
des Wahlrechts noch in der 16. Wahlperiode schied deswegen letzten Endes aus. Während die SPD und die Grünen eine Lösung anstrebten, die das negative Stimmengewicht durch eine Verrechnung anfallender interner Überhänge mit anderen nicht überhängenden Landeslisten ausgeschlossen hätte, spielte die CDU/CSU auf Zeit. ${ }^{35}$ Eingedenk der Tatsache, dass Union und SPD in der Großen Koalition gemeinsam abstimmen mussten, hatte die CDU/CSU die strategisch günstigere Position. Zusätzliche Überhangmandate versprachen eine knappe Mehrheit der Union mit der FDP komfortabler werden zu lassen oder eine knapp verfehlte Zweitstimmenmehrheit durch die Überhangmandate zu einer parlamentarischen Mehrheit werden zu lassen. ${ }^{36}$

\section{Wablrechtdebatte nach 2009}

Die Bundestagswahl 2009 bestätigte insoweit die Erwartungen der Union, dass ihr in erheblichem Umfang Überhangmandate zufielen. Mit 21 internen für die CDU und 3 externen Überhangmandaten für die CSU vergrößerte die Koalition ihre freilich ohnehin bestehende Mehrheit beträchtlich. Wenngleich Überhangmandate aus einem Bündel verschiedener Faktoren entstehen, ${ }^{37}$ hat bei dieser Wahl die massive Schwäche der SPD und das auffällige Splittingverhalten der FDP-Wähler zugunsten der Union ${ }^{38}$ letztlich die Verluste von CDU und CSU in der Wählergunst bei der Mandatszuteilung mehr als kompensiert. Obwohl die CDU und die CSU 1,4 Prozentpunkte verloren haben, können die Unionsparteien dank der Überhangmandate statt 226 Abgeordnete nun 239 in den Bundestag entsenden.

Die möglich gewordene Koalition von Union und FDP führt aber dazu, dass zahlreiche Reformvorschläge zur Beseitigung des negativen Stimmengewichts, die in der wissenschaftlichen Debatte vorgebracht wurden, von vornherein zum Scheitern verurteilt

35 Jesse (»Verhältniswahl und Gerechtigkeit«aaO. (FN 1), S. 126) unterstellt auch der SPD lange auf Zeit gespielt zu haben und führt deren Bereitschaft zu einer Reform wie auch Decker (Regieren im "Parteienbundesstaat". Zur Architektur der deutschen Politik, aaO. (FN 10), S. 154) auf für sie nachteilige Modellrechnungen im Lichte der Umfragen von 2009 zurück. Die von Decker dabei angeführten Modellrechnungen lagen allerdings erst im Juni des gleichen Jahres vor, wiewohl sich die SPD schon im Februar auf eine Reform noch vor der Bundestagswahl 2009 festgelegt hatte (Thomas Kröter, »Mehr als nur ein Schönheitsfehler « in: Frankfurter Rundschau vom 12.2.2009; Holger Schmale, »SPD und Grüne wollen das Wahlrecht reformieren « in: Berliner Zeitung vom 12.2.2009). Allerdings war schon länger bekannt, dass Überhangmandate prinzipiell eine Umkehrung der Mehrheitsverhältnisse bewirken könnten (Behnke, »Überhangmandate: Ein (behebbarer) Makel im institutionellen Design des Wahlsystems « aaO. (FN 21), S. 1255).

36 »Überhangmandate stärken Schwarz-Gelb« in: Der Spiegel, 27/2009 vom 29.6.2009; Jesse, »Verhältniswahl und Gerechtigkeit« aaO. (FN 1), S. 126.

37 Jesse, »Verhältniswahl und Gerechtigkeit«aaO. (FN 1), S. 119; Behnke, »Ein integrales Modell der Ursachen von Überhangmandaten« aaO. (FN 25); Behnke, Das Wablsystem der Bundesrepublik Deutschland. Logik, Technik und Praxis der Verbältniswahl, aaO. (FN 4), S. $182 \mathrm{ff.}$

38 Meyer, Die Zukunft des Bundestagswablrechts. Zwischen Unverstand, obiter dicta, Interessenkalkül und Verfassungsverstoß, aaO. (FN 30), S. $72 \mathrm{ff}$. 
sind. Die Einführung eines Grabenwahlrechts ${ }^{39}$ oder eines reinen Mehrheitswahlrechts $^{40}$ würden sicher am Widerstand der FDP scheitern. Die Einführung von mehrheitsfördernden Prämien ${ }^{41}$ dürfte rechtlich heikel und politisch in einer kleinen Koalition ebenfalls nicht durchsetzbar sein. ${ }^{42}$ Letzteres wäre zudem für die Union unannehmbar, weil bei konsequenter Umsetzung dann in der Regel die SPD begünstigt wäre, da CDU und CSU ja als zwei Parteien bei den Wahlen antreten. ${ }^{43}$ Möglich wäre ein Wahlsystemwechsel hin zu einem mehrheitsbildenden Wahlrecht wohl nur in der Großen Koalition gewesen und dann wohl auch nur, wenn es dauerhaft zur Großen Koalition keine alternative Mehrheitsbildung gegeben hätte. ${ }^{44}$ Dazu kam es aber nicht und dazu bestand auch - wie gezeigt - keine Veranlassung.

In einer Koalition sind aber auch weniger weitreichende Reformvorschläge unrealistisch, die potentiell zum Nachteil eines Koalitionspartners wirken. Deswegen scheidet auch eine Aufgabe des $Z$ weistimmenwahlrechts ${ }^{45}$ aus, weil die FDP hiervon mutmaßlich in besonderem Maße profitiert hat. Ferner sind Vorschläge kaum Erfolg versprechend, die Überhangmandate dadurch kappen wollen, in dem die Zahl der Direktmandate einer Partei auf den Proporzanspruch im jeweiligen Bundesland begrenzt wird. Der relativ am schlechtesten abschneidende Direktkandidat würde dabei das eigentlich gewonnene Mandat verfehlen. ${ }^{46}$ Alternativ beschränkt man den drohenden Mandatsverlust auf Wahlkreise, in denen keine andere Partei ein Listenmandat errungen hat. ${ }^{47}$ In beiden Fällen wäre aller Voraussicht nach die CSU latent betroffen.

Es zeigt sich also, dass die Struktur des Parteiensystems und die daraus resultierende Regierungsbildung durchaus Einfluss auf die Struktur des Wahlsystems haben. Jedenfalls

39 Strohmeier, »Vergangene und zukünftige Reformen des deutschen Wahlsystems «aaO. (FN 15), S. 31; siehe auch Josef Isensee, »Funktionsstörungen im Wahlrecht: Das negative Stimmgewicht. Denkbare Lösungen eines Dilemmas «, in: Das Deutsche Verwaltungsblatt, 5/2010, S. 269-277.

40 Hans Herbert von Arnim, »Mehrheitswahl und Partizipation« in: Gerd Strohmeier (Hg.), Wablsystemreform, Baden-Baden 2009, S. 183-210.

41 Nohlen, Wablrecht und Parteiensystem, Zur Theorie und Empirie der Wablsysteme, aaO. (FN 7), S. 357; Dieter Nohlen, »Erfolgswertgleichheit als fixe Idee oder: Zurück zu Weimar? Zum Urteil des Bundesverfassungsgerichts über das Bundeswahlgesetz vom 3. Juli 2008 « in: Zeitschrift für Parlamentsfragen, 1/2009, S. 179-195.

42 Meyer, Die Zukunft des Bundestagswablrechts. Zwischen Unverstand, obiter dicta, Interessenkalkül und Verfassungsversto $\beta$, aaO. (FN 30), S. 30.

43 Lediglich 1953, 1957, 1990 und 2009 war die CDU die relativ stärkste Partei.

44 Grotz, »Verhältniswahl und Regierbarkeit« aaO. (FN 15), S. 165; Shirvani, Das Parteienrecht und der Strukturwandel im Parteiensystem, aaO. (FN 7), S. $451 \mathrm{f}$.

45 Meyer, Die Zukunft des Bundestagswablrechts. Zwischen Unverstand, obiter dicta, Interessenkalkül und Verfassungsverstoß, aaO. (FN 30); Eckhard Jesse, »Reformvorschläge zur Änderung des Wahlrechts", in: Aus Politik und Zeitgeschichte, B 52/2003, S. 3-11, hier: S. 11; in ähnlicher Weise Prittwitz, »Vollständig personalisierte Verhältniswahl «, aaO. (FN 3), S. 19.

46 Ebd., S. 83ff.; grundlegend dagegen Isensee, »Funktionsstörungen im Wahlrecht: Das negative Stimmgewicht. Denkbare Lösungen eines Dilemmas«aaO. (FN 39), S. 274.

47 Friederike Oelbermann / Friedrich Pukelsheim / Matthias Rossi / Olga Ruff, »Eine schonende Verbindung von Personen- und Verhältniswahl zum Abbau negativer Stimmgewichte bei Bundestagswahlen" in: Kritische Vierteljabresschrift für Gesetzgebung und Rechtswissenschaft, 1/2011 (94. Jg.) S. 56-80. 
lässt sich nicht leugnen, dass die spezifischen Interessen der Parteien an einem Fortbestand des Status Quo immens sind. Bedingt durch das Wahlergebnis besteht die Unionsfraktion zudem gegenwärtig fast vollständig aus direkt gewählten Bundestagsabgeordneten. Jede Reform, die deren Wiederwahlchancen schmälern würde, wäre somit schwerlich durchzusetzen, zumal eine solche Reform ja unmittelbare Wirkung für die folgende Legislaturperiode entfaltet.

Dementsprechend muss auch der auf den ersten Blick originelle Vorschlag verworfen werden, das Problem einfach zu minimieren, indem die Zahl der Wahlkreise halbiert und darin die beiden bestplatzierten Kandidaten direkt gewählt werden. ${ }^{48}$ Etliche Abgeordnete müssten auf ihr Direktmandat verzichten und sich allein auf das unsichere Rennen bei den Listenplätzen einlassen, bei dem wiederum wahrscheinlich die Inhaber von $\mathrm{Di}$ rektkandidaturen bevorzugt würden. Damit könnten die von Union und SPD dann aufgestellten Wahlkreiskandidaten in aller Regel bereits ihrer Wahl in den Deutschen Bundestag sicher sein. Die meisten Wahlkreise würden nämlich tote Rennen um die Direktmandate erleben. In gerade einmal einem Zehntel der Wahlkreise gibt es überhaupt nur ein enges Rennen zwischen dem zweit- und drittplatzierten Bewerber. Dass eine Partei fernerhin das Risiko eingeht und mit zwei Kandidaten antritt, um eventuell beide Mandate zu gewinnen, dürfte ebenfalls unwahrscheinlich sein. Sollte sich zudem die gegenwärtige demoskopische Stärke der Grünen verfestigen, könnten zudem leicht Überhangmandate für die vorwiegend zweitplatzierte Partei anfallen, woraus dann wiederum negativen Stimmengewichte resultieren könnten.

Gleichzeitig ist klar, dass das bestehende Wahlrecht verfassungswidrig ist, womit eine Änderung unausweichlich ist. Die Suche nach einer »minimalinvasiven Lösung « ${ }^{49}$ wird daher angestrebt. Realistischerweise können nur solche Lösungen umgesetzt werden, die den Interessen der zahlreichen direkt gewählten Abgeordneten bei der Union wie den Interessen der FDP als Partei gerecht werden. Es gibt somit deutliche Hinweise darauf, dass das Parteiensystem also nun das Wahlsystem strukturiert.

\section{Realistische Lösungsansätze}

Im Grunde besitzen nur vier Reformvorschläge eine Chance auf politische Realisierbarkeit: Die Trennung der Listenverbindungen, die Verrechnung der Überhangmandate mit Listenmandaten, die Einführung von Ausgleichsmandaten oder eine getrennte Mandatszuteilung je Bundesland.

Systemimmanent wäre die wohl einfachste Lösung, die Verbindungen der Landeslisten in $\$ 7 \mathrm{BW}$ ahlG aufzugeben und eine Listenverbindung allein bei der Ermittlung der Sperrklausel vorzunehmen. Die Landeslisten der zuteilungsberechtigten Parteien würden also sämtlich um die Mandate zum Deutschen Bundestag miteinander konkurrieren.

48 Behnke, Das Wablsystem der Bundesrepublik Deutschland. Logik, Technik und Praxis der Verbältniswabl, aaO. (FN 4), S. $225 \mathrm{ff}$.

49 Daniel Lübbert, »Negative Stimmengewichte bei der Bundestagswahl 2009 « in: Zeitschrift für Parlamentsfragen, 2/2010, S. 278-289, hier: S. 289. 
Interne Überhangmandate, die sich aus dem bloßen Zusammenspiel von Ober- und Unterverteilung ergeben, entfielen. Der vom Verfassungsgericht problematisierte Effekt, wonach eine Partei sich selbst schaden kann, wenn sie mehr Stimmen in einem Land erhält, in dem sie Überhangmandate erzielt oder erzielen kann, wird freilich nicht ganz beseitigt, sondern nur transformiert. Es kann durchaus sein, dass sich der Mandatsgewinn in Land A bei Partei X zu Lasten derselben Partei in Land B auswirkt. Das muss allerdings nicht zwingend so sein. Zweifelsfrei käme eine solche Situation aber seltener als bislang vor.

Das Verfahren birgt aber einen gravierenden Nachteil in sich. Es entfiele die so genannte Reststimmenverwertung. Hierbei handelt es sich um den Sachverhalt, dass die Landesliste einer Partei in aller Regel immer einen etwas höheren Mandatsanspruch erzielen kann, als ihr an ganzzahligen Mandaten beim Abrunden des optimalen Verhältnisergebnisses zustünde. Der nicht in Mandate übersetzte Rest wird bis auf einen unvermeidlichen Rest, der kleiner als der Grundwert eines halben Mandats ist, gewöhnlich mit den übrigen Reststimmen auf der Oberverteilung berücksichtigt. Dies entfiele bei einer Lösung der Listenverbindungen. Sofern nicht länderübergreifende Listenwahlkreise gebildet werden ${ }^{50}$ und sich Auf- und Abrundungen der Mandatsverteilung nicht ausgleichen, kann es im äußersten Extremfall sein, dass eine Partei dadurch in jedem Bundesland die Zuteilung eines weiteren Mandats knapp verpasst. In jedem der 16 Bundesländer könnte eine Partei somit jeweils ein halbes Mandat verpassen, weswegen in der Summe knapp 8 Mandate fehlen, die anderenfalls auf der Oberverteilung sicher entstanden wären. Auf der Grundlage des 2009 anzuwendenden Divisors entspräche das bis zu 547.000 Stimmen, die keine Wirkung entfaltet hätten.

Dieser Extremfall relativiert sich im Lichte der tatsächlichen Wahlergebnisse, weil Abrundung und Aufrundungen sich in der Regel knapp die Waage halten. Bei der Bundestagswahl 2009 hätten konkret SPD und CSU ein Mandat weniger erhalten (dafür aber jeweils ein weiteres Überhangmandat erzielt), wohingegen Grüne und FDP jeweils ein weiteres Mandat bekommen hätten. Rechnerisch wären die Unwuchten also eher gering, aber dennoch vorhanden.

Obwohl dieses Verfahren am bisherigen Zuteilungsverfahren bis auf die Unwucht bei der Reststimmenverwertung wenig verändern würde, findet es nur wenige Anhänger. Insbesondere die kleineren Parteien sind potentiell eher durch die fehlende Reststimmenverwertung betroffen als die größeren Parteien. Vor allem in Bremen, Saarland, Mecklenburg-Vorpommern und auch Hamburg erhalten die Stimmen von FDP, Grünen und Linken ohnehin oftmals nur über die Reststimmenverwertung eine Wirkung bei der Mandatszuteilung. Demgegenüber können Union und SPD die fehlende Reststimmenverwertung eher verschmerzen, weil sie den Verlust teilweise durch Überhangmandate kompensieren.

50 Isensee, »Funktionsstörungen im Wahlrecht: Das negative Stimmgewicht. Denkbare Lösungen eines Dilemmas«aaO. (FN 39), S. 275. 
Die Überhangmandate sind es aber nun, die einen großen Teil der Kritik auf sich ziehen. ${ }^{51}$ Diese blieben bei einer Trennung der Listenverbindungen in vergleichbarem Umfang erhalten beziehungsweise könnten - wie gezeigt - sogar ansteigen. Überhangmandate fallen dabei aber wie im bisherigen System nicht an, weil eine Partei besonders gute Direktkandidaten aufbieten kann, sondern sie sind Ausdruck der Schwäche der beiden Volksparteien in Verbindung mit einer besonderen regionalen Hochburgenbildung. ${ }^{52}$

Deswegen verwundert es nicht, dass die Grünen wie auch die Linken eine Verrechnung interner Überhangmandate mit Listenmandaten in nicht überhängenden Ländern erneut ins Spiel gebracht haben. Dieser Ansatz entspricht im Wesentlichen dem Augsburger Zuteilungsverfahren nach Friedrich Pukelsheim ${ }^{53}$ und ist technisch recht einfach zu realisieren. Allerdings gibt es in der Zwischenzeit einige Länder, in denen regelmäßig Überhangmandat auftreten, und einige Länder, die keine Überhänge erwarten lassen. Letztere müssten dann für die Kompensation herhalten und würden damit eine strukturelle Schwächung ihres Einflusses in Kauf nehmen. Zudem gibt es einige systematische Probleme. Die Verrechnung über die Landeslisten hinweg bevorteilt die CSU, weil diese sich mit keiner anderen Landesliste verrechnen müsste und ihr im Zweifelsfalle als einziger Partei somit Überhänge erhalten blieben.

Erschwerend kommt hinzu, dass bei der CDU das letzte Mal eine Reihe prominenter Vertreter (von der Leyen, Lammert, von Klaeden, Bergner, Hintze, Neumann) nur über die Landesliste eingezogen waren. Wären bei der Bundestagswahl 2009 bereits die angefallenen Überhänge verrechnet worden, hätte kein einziger dieser Abgeordneten ein Mandat erzielt.

Der dritte realistische Reformvorschlag ist eine auf dem Augsburger Zuteilungsverfahren aufbauende, aber im Ergebnis stärker an das bisherige System angelehnte Mandatsberechnung. ${ }^{54}$ Demnach folgt auf Ober- und Unterverteilung eine Korrektur der Verteilung, indem die Zahl der Mandate auf der Oberverteilung so lange erhöht wird, bis die Zahl der Mandate der überhängenden Parteien mit dem Proporzergebnis übereinstimmt. Anders als bei einem vollständigen proportionalen Ausgleich von Überhang-

51 Behnke, »Überhangmandate: Ein (behebbarer) Makel im institutionellen Design des Wahlsystems « aaO. (FN 21); Daniel Lübbert / Felix Arndt / Friedrich Pukelsheim, »Proporzwahrende Anpassung der Bundestagsgröße - ein Lösungsvorschlag für das Problem der negativen Stimmgewichte bei Bundestagswahlen «, http://opus.bibliothek.uni-augsburg.de/volltexte/ 2011/1706/pdf/ mpreprint_11_002.pdf (Stand: 7.3.2011); dagegen Pappi / Herrmann, »Überhangmandate ohne negatives Stimmgewicht: Machbarkeit, Wirkungen, Beurteilungen« aaO. (FN 15).

52 Behnke, »Überhangmandate: Ein (behebbarer) Makel im institutionellen Design des Wahlsystems « aaO. (FN 21).

53 Pukelsheim, »Bundeswahlgesetz - nächste Etappe« aaO. (FN 31); im Ergebnis vergleichbar der Vorschlag einer virtuellen Bundesliste (Behnke, »Überhangmandate: Ein (behebbarer) Makel im institutionellen Design des Wahlsystems« aaO. (FN 21), S. 1260ff.).

54 Lübbert / Arndt / Pukelsheim, »Proporzwahrende Anpassung der Bundestagsgröße - ein Lösungsvorschlag für das Problem der negativen Stimmgewichte bei Bundestagswahlen« aaO. (FN 51). 
mandaten ${ }^{55}$ hält sich die Zahl der Ausgleichsmandate in vergleichsweise engen Grenzen. Die Zahl der Überhangmandate würde im Ergebnis allein durch Ausgleichsmandate so weit kompensiert werden, dass ein negatives Stimmgewicht ausgeschlossen wäre. Sofern mehr als eine Partei Überhangmandate erzielt, würde zunächst die am stärksten überhängende Partei ausgeglichen werden. Im Zuge dessen würden die Überhänge der weiteren überhängenden Parteien idealerweise bereits mit den anfallenden Ausgleichsmandaten verrechnet werden. Als problematisch stellen sich hierbei die Überhangmandate der CSU dar. Sofern SPD oder CDU deutlich mehr Überhänge als die CSU produzieren würden (was bislang stets der Fall war), wäre damit ein Ausgleich der CSU-Überhänge bereits erledigt. Anderenfalls entfalten die CSU-Mandate jedoch eine immense Hebelwirkung hinsichtlich der erforderlichen Ausgleichsmandate.

2009 hätte ein CDU-Überhangmandat circa zwei Ausgleichsmandate nach sich gezogen. Ein Überhangmandat der CSU hingegen würde, sofern keine weitere Partei Überhangmandate erzielt, aber ungefähr 13 Ausgleichsmandate erzeugen. Dieses Problem ließe sich jedoch heilen, indem zwischen externen und internen Überhangmandaten differenziert wird. So dann könnte bei externen Überhangmandaten auf einen Ausgleich verzichtet werden. Damit bliebe die Hebelwirkung begrenzt auf potentielle Überhangmandate der Linken, die bislang noch nicht aufgetreten sind. Alternativ böte sich noch der Ausgleich allein in dem betreffenden Bundesland an. ${ }^{56}$ Dieses brächte allerdings den Nachteil mit sich, dass dadurch dasjenige Land, welches durch die Überhänge ohnehin schon mehr Mandate erhielte noch einen weiteren Bonus bekäme.

Trotz des noch offenen CSU-Problems kann sich insbesondere die SPD für diese Art des Verrechnungsverfahrens erwärmen. Ihr geht es vorrangig um die Überhangmandate, obwohl sie ja zwischenzeitlich selbst davon stark profitiert hat. Allerdings zeigen verschiedene Berechnungen eben, dass die Überhangmandate eigentliche Mehrheiten umkehren können. Dabei ist gegenwärtig vor allem die Gefahr gegeben, dass so eine CDU/ CSU/FDP-Minorität durch perfektes lagerimmanentes Splitting zu einer Mehrheit werden könnte beziehungsweise eine knappe rot-grüne Mandatsmehrheit darüber verhindert würde. Mithin würden die strategischen Optionen der SPD geringer werden, weil deren Lager nicht derart handlungsfähig ist ${ }^{57}$ und damit die Wähler des potentiellen Lagers auch weniger zu strategisch entsprechendem Handeln verleitet sind. Während man das negative Stimmengewicht als akademisches Problem mit nur geringen Effekten ansehen kann, stellen die Überhangmandate sowohl in ihrem Volumen als auch hinsichtlich der drohenden Umkehrung tatsächlicher Mehrheiten ein veritables Problem für die Ak-

55 Friedrich Pukelsheim / Sebastian Maier, »Parlamentsvergrößerung als Problemlösung für Überhangmandate, Pattsituationen und Mehrheitsklauseln « in: Zeitschrift für Parlamentsfragen, 2/2008, S. 312-322; siehe auch Lübbert, »Negative Stimmengewichte bei der Bundestagswahl 2009« aaO. (FN 49), S. $287 \mathrm{f}$.

56 Ebd., S. 289.

57 Karl-Rudolf Korte: »Parteienwettbewerb. Wählen und Regieren im Schatten der Großen Koalition « in: David Gehne / Tim Spier (Hg.), Krise oder Wandel der Parteiendemokratie, Festschrift für Ulrich von Alemann, Wiesbaden 2010, S. 121-131, hier S. 123. 
zeptanz des Wahlrechts dar. ${ }^{58}$ So hat das Bundesverfassungsgericht schon in seiner maßgeblichen Entscheidung von 1997 darauf hingewiesen, dass Überhangmandate nur bis zu einer bestimmten Größenordnung tolerabel seien, ohne das näher zu spezifizieren. In der rechtswissenschaftlichen Literatur ${ }^{59}$ wird auf die 5\%-Sperrklausel als analoge Größenordnung verwiesen, die 2009 fast erreicht worden ist. Insofern ist das vom ersten parlamentarischen Geschäftsführer der SPD-Fraktion, Thomas Oppermann, postulierte Ziel, den »Wildwuchs « bei den Überhangmandaten ${ }^{60}$ zu begrenzen, ein machtpolitisch legitimes Interesse, welches aus den gleichen Erwägungen heraus von der Union genau andersherum eingeschätzt wird. Offensichtlich beeinflussen die aus dem Parteiensystem resultierenden Machtoptionen gegenwärtig eher das Wahlsystem als umgekehrt.

Somit gerät als viertes eine Lösung ins Visier, die das Auftreten eines negativen Stimmengewichts ausschließt, Überhänge aber nicht vermeidet. Dieses wäre mit einer separaten Mandatszuteilung in jedem einzelnen Bundesland möglich. ${ }^{61}$ Im Prinzip erinnert das an die bereits erwähnte Auflösung der Listenverbindungen. Anfallende Überhangmandate würden eindeutig und durchgängig externer Natur sein und damit frei vom Effekt des negativen Stimmengewichts. Anders als bei der Lösung der Listenverbindung wäre die Mandatszuteilung im jeweiligen Bundesland aber nicht mehr vom Ausgang der Wahl in den übrigen Bundesländern abhängig, sofern die Länder vorab ein festes Mandatskontingent zugewiesen bekämen.

Wenn man zugleich aber den Anspruch wahrt, dass in jedem Teilwahlgebiet hälftig Listen- und Wahlkreismandate vergeben werden, führt das in Verbindung mit den verschiedenen Wahlbeteiligungen dazu, dass es in jedem Land sehr unterschiedliche Divisoren für die Berechnung der Mandatszuteilung wirken. Die Spanne reicht im Lichte aktueller Umfragen und gewöhnlicher Wahlbeteiligungen gegenwärtig von ca. 55.000 (Mecklenburg-Vorpommern) bis zu 80.000 Stimmen (Bremen). Berücksichtigt man dieses, existieren zusätzliche natürliche Sperrklauseln ${ }^{62}$ von über $14 \%$ in Bremen und von über 7,5\% im Saarland, die neben der weiterhin bundesweit wirksamen $5 \%$-Klausel zu überwinden wären. Wenn zudem die bundesweite Sperrklausel nicht mehr aufrecht erhalten werden könnte, würde eine länderbezogene Anwendung das Problem aufwerfen, dass es gemessen an der Zahl der absoluten Stimmen im bevölkerungsreichsten Bundesland Nordrhein-Westfalen bedeutend schwieriger wäre, ein Mandat zu erlangen als im

58 Pukelsheim, »Erfolgswertgleichheit der Wählerstimmen zwischen Anspruch und Wirklichkeit« aaO. (FN 31).

59 Meyer, Die Zukunft des Bundestagswablrechts. Zwischen Unverstand, obiter dicta, Interessenkalkül und Verfassungsverstoß, aaO. (FN 30), S. 37, das Urteil selbst siehe BVerfGE 95, 335.

60 Thomas Oppermann, »Zu Risiken und Nebenwirkungen von Überhangmandaten « in: Frankfurter Allgemeine Zeitung vom 7.1.2011.

61 »Vorschlag zur Wahlrechtsreform« in: Frankfurter Allgemeine Zeitung vom 30.9.2010; Pappi / Herrmann, »Überhangmandate ohne negatives Stimmgewicht: Machbarkeit, Wirkungen, Beurteilungen « aaO. (FN 15).

62 Friedrich Pukelsheim / Sebastian Maier / Peter Leutgäb, Zur Vollmandats-Sperrklausel im Kommunalwahlgesetz, in: Nordrhein-Westfälische Verwaltungsblätter, Zeitschrift für öffentliches Recht und Verwaltung, Heft 3/2009, S. 85-90, hier S. 87. 
kleinsten Land Bremen. ${ }^{63}$ Mit dem vom Verfassungsgericht hoch gehaltenen Erfolgswert der Stimmen wäre das jedenfalls schwerlich in Einklang zu bringen.

Obgleich eine Umrechnung des Bundestagswahlergebnisses von 2009 auf ein solches Modell nur geringe Abweichungen in der Mandatszuteilung zeigt, wären gewisse Einflüsse auf die Parteienstruktur zu erwarten. Wegen der hohen Sperrklauselwirkung könnten die kleineren Parteien den Wahlkampf in einigen Bundesländern von vornherein aufgeben. Mit anderen Worten: In Bremen findet keine FDP-Großveranstaltung mehr statt und im Saarland verzichten die Grünen auf jeden professionellen Rednereinsatz.

Ferner ist bei einer solchen Lösung klar, wie ein Wähler abstimmen muss, wenn er seiner gewünschten Parteikonstellation nutzen will. Bislang konnten die Wähler das nur mit einer gewissen Wahrscheinlichkeit einkalkulieren. Bei einer separaten Zuteilung der Mandate nach Bundesländern kann er hingegen weitaus verlässlicher taktisch wählen. Pappi und Herrmann versprechen sich genau davon, dass die Lagerbildung schon vor der Bundestagswahl klar zum Ausdruck kommt. ${ }^{64}$

Die Wähler könnten in denjenigen Ländern, in denen ein Überhangmandat wahrscheinlich ist, aber auch ein hiervon unabhängiges zweifach doppeltes Stimmgewicht erzielen. Im Gegensatz zum jetzigen Wahlrecht würde beim Splitting diese Wahlentscheidung niemals zu Lasten ihrer eigentlichen Parteipräferenz gehen, weil die ausbleibende Stimme für die eigentliche Erstpräferenz ja nicht mehr bei der Oberverteilung fehlt. Zum anderen kann durch das zielgerichtete Splitting ein Überhang entstehen, der das Gewicht des Bundeslands im Bundestag erhöht. Das bedeutet, ein derart taktisch wählender Bürger kann selbst durch die bewusste Wahl des politischen Gegners mit der Erststimme (oder unter Umständen auch mit der Zweitstimme) zielgerichtet das Stimmengewicht seines Bundeslandes maximieren. Das käme insbesondere dann zum Tragen, wenn die eigentliche Parteipräferenz keine Chance auf ein Direktmandat hat. Diejenigen Wähler, die also bewusst das Splitting einsetzen, können im Ergebnis den Wert ihrer Stimme zu Lasten derjenigen erhöhen, die treu beide Stimmen ihrer eigentlichen Parteipräferenz geben.

Eigentlich will das Wahlrecht verhindern, dass einzelne Wähler ihr Stimmenpotential durch Taktik erhöhen. Bei den Überhangmandaten wird dies noch toleriert. Bei den marginalen Berliner Zweitstimmen wurde es hingegen politisch nicht hingenommen. Hierbei handelt es sich um diejenigen Wähler der PDS, die mit ihrer Erststimme 2002 die beiden siegreichen Kandidatinnen gewählt haben, mit der Zweitstimme aber eine andere Partei. Hätte es sich um erfolgreiche Einzelbewerber gehandelt, hätten die Zweitstimmen dieser Wähler wegen \6 I 2 BWahlG nicht gezählt. Weil aber eine unerfolgreiche PDS-Landesliste existierte, kam der entsprechende Passus nicht zur Anwendung. Zwischen den Bundestagsparteien gibt es mittlerweile einen Konsens, dass dies auch im Falle von erfolgreichen Direktkandidaturen von Parteien der Fall sein soll, die nicht die Sperrklausel überwunden haben. Der Hintergrund ist, dass jeder Wähler im Ergebnis mit nur einer Stimme Einfluss auf die Wahl des Bundestags haben soll. Wenn aber der

63 Jesse, Demokratie in Deutschland. Diagnosen und Analysen, aaO. (FN 16), S. 224.

64 Ebd., S. 272.

ZfP 58. Jg. 3/2011 
Direktbewerber bereits erfolgreich war, dessen Partei aber nicht, dann hätte der betreffende Wähler noch eine zweite Stimme, die er ebenfalls nutzbringend einsetzen kann. Während das doppelte Stimmgewicht bei der Berliner Zweitstimme beseitigt werden soll, käme es einer Aufteilung des Wahlgebiets wieder hervor und würde allen schlauen Taktikern Tür und Tor öffnen, um ihren Einfluss zu verdoppeln.

Während die ersten drei Modelle den Interessen einzelner Koalitionspartner zuwiderliefen, sind die Schönheitsfehler im letzten Modell doch immens. Die Koalition steuert gleichwohl auf dieses System zu. Sie will allerdings zugleich den Effekt mit den unterschiedlichen Erfolgswerten nivellieren und die Sperrklausel zwingend bundesweit anwenden. Während ersteres aus Gründen der verfassungsrechtlich wichtigen Erfolgswertgleichheit leicht nachzuvollziehen ist, resultiert letzteres eher aus einem Kalkül, das auf das Parteiensystem zurückverweist. Wenn die Sperrklausel nur noch je Bundesland Anwendung finden kann, so würde einerseits die FDP latent Gefahr laufen, diese in einigen Ländern zu verfehlen, und andererseits eine Partei wie die NPD die Chance besitzen, darüber wenigstens einige Mandate zu erlangen. Hinzu kommt noch, dass es Hinweise gibt, dass es beim Bundestag als unitarischem Organ des gesamten deutschen Volks überaus schwierig wäre, dieses als Gesamtheit von 16 Teilvölkern zu konstruieren. ${ }^{65}$

Also ersann sich die Koalition ein neues Modell. Im Ergebnis wurde aber nicht das vierte Modell wirklich systematisch weiterverfolgt, sondern die Mechanismen von Oberund Unterverteilung des bestehenden Wahlrechts einfach vertauscht. In einem ersten Schritt sollen die Gesamtmandate auf die einzelnen Bundesländer anhand der Wählerzahl verteilt werden und dann sollen diese auf die einzelnen Parteien in jedem Land zugeteilt werden. ${ }^{66}$ Zuteilungsberechtigt sollen dabei Parteien sein, die bundesweit eine der beiden Sperrklauseln überwunden haben.

Insoweit die Zuteilung der Proporzmandate auf die Länder vorab an Hand der Wahlbeteiligung erfolgt, ist das negative Stimmengewicht nicht mehr ausgeschlossen. Es wird allerdings zufälliger und absurder als bislang. Mehr Stimmen für eine Partei A im Land $\mathrm{X}$ bringen nämlich nicht der Partei A zwingend einen Nutzen, sondern zunächst einmal bloß dem Land X einen Mandatsgewinn zulasten von Land Y. Sofern die zusätzlichen Stimmen bei A nicht ausreichen, damit $\mathrm{A}$ auch bei der Verteilung in $\mathrm{X}$ dieses zusätzliche Mandat erringt, profitiert davon dann Partei B im Land X. Wenn sich nun der so realisierte Gewinn eines weiteren Mandats bei Partei B zulasten eines Mandats von Partei A in Land Y realisiert, dann würde sich der Effekt des negativen Stimmengewichts im Prinzip verdoppeln. Hatte im bisherigen Wahlrecht eine Stimme für A nur bei Vorliegen von Überhangmandaten für A im Land X eine geringere Mandatszuteilung für A im Land Y zur Folge gehabt, so hätte nunmehr eine Stimme für eine Partei nicht nur eine geringere

65 Hans Meyer, »Lösungsmöglichkeiten nach dem Wahlrechtsurteil des BVerfG vom 3. Juli 2008 « in: Das Deutsche Verwaltungsblatt, 3/2009, S. 137-146.

66 Bundestagsdrucksache 17/6290; Florian Kain / Christian Mutter, »Wahlrechtsänderung im Alleingang « in: Die Welt vom 9.4.2011; Sebastian Fischer, »Koalition ringt um neues Wahlrecht in: die tageszeitung vom 20.4.2011. 
Mandatszuteilung für A in Y zur Folge, sondern könnte zugleich einen Mandatszuwachs für B in X bewirken. Neben dem negativen Stimmengewicht wären weitere eigenartige Effekte zu erwarten. So kann nämlich ein Stimmenzuwachs von Partei A in X auch zu einem Mandatsverlust bei Partei C in Y führen und zugleich einen Mandatsgewinn von Partei B in X bewirken. Der Effekt der Wahlstimme würde also »verzufallt « werden. Das aber kann kaum der Intention des Verfassungsgerichts entsprechen, wonach eine Stimme für eine Partei auch eine Wirkung für selbige prinzipiell entfalten können muss.

Das Ganze würde obendrein sehr regelmäßig anfallen. Bei der Bundestagswahl 2009 wäre jedenfalls die Zahl der Fälle von negativem Stimmengewicht nicht gesunken. Problemlos lassen sich etwa die folgenden Beispiele ermitteln, die in ihrer Qualität dem negativen Stimmengewicht entsprächen:

- 17.500 Stimmen mehr für die CDU in Baden-Württemberg hätten der SPD in BW ein Mandat,

- 30.000 Stimmen mehr für die CDU in Sachsen hätten der FDP in Sachsen ein zusätzliches Mandat und

- 50.000 Stimmen mehr für die CDU in Hamburg hätten der SPD dort ein weiteres Mandat beschert.

- Je 50.000 Stimmen mehr für die CDU in Hessen und in Sachsen würden der SPD ein Mandat mehr in Hessen und der FDP ein Mandat mehr in Sachsen bescheren.

- 10.000 Stimmen für die FDP in Brandenburg hätten der SPD einen Mandatsgewinn eingebracht.

- 50.000 Stimmen weniger für die Linke in Niedersachsen hätten der Partei ein zusätzliches Mandat in Nordrhein-Westfalen beschert.

In allen hier aufgeführten Fällen hätte die CSU ein Mandat abgeben müssen, das sie aber mit einem weiteren Überhangmandat kompensiert hätte. Im vierten Beispiel hätte zudem die SPD in Nordrhein-Westfalen ein Mandat abgegeben. Ein wirklicher Fall von negativem Stimmengewicht lässt sich also hierbei nur für den Fall der Linken konstruieren, es sei denn man rechnet CDU und CSU zusammen.

Gerade das Beispiel mit der Linken zeigt zudem, dass der Effekt des negativen Stimmengewichts dadurch unabhängig von Überhangmandaten auftreten könnte. Der alte Merksatz, wonach Überhangmandate eine notwendige, aber keine hinreichende Bedingung für negatives Stimmengewicht sind ${ }^{67}$ wäre mit diesem Modell widerlegt. Aus wissenschaftlicher Sicht eine sicherlich begrüßenswerte Falsifizierung. Aus politisch-rechtlicher Bewertung würden aber so die Vorgaben des Bundesverfassungsgerichts klar verfehlt werden.

Fraglich ist zudem, ob eine Verteilung der Mandate auf die Bundesländer anhand der Wahlbeteiligung überhaupt verfassungskonform ist. Der unitarische Charakter des Bundesvolkes würde möglicherweise eher gewahrt bleiben als bei einer Vorabberechnung über die Bevölkerung. Dem steht entgegen, dass gegenüber dem bisherigen Modell die-

67 Meyer, Die Zukunft des Bundestagswablrechts. Zwischen Unverstand, obiter dicta, Interessenkalkül und Verfassungsverstoß, aaO. (FN 30), S. 59; Pehle, »Die Bundestagswahlen von 2005 und 2009: Verfassungswidrig und doch gültig?« aaO. (FN. 20), S. 474. 
jenigen Länder ihre Repräsentation erhöhen würden, in denen viele Wähler einer Partei ihre Stimme geben, die von der Mandatszuteilung ausgeschlossen bleibt, weil sie die Sperrklausel verfehlt hat.

Tabelle 1: Auswirkung Koalitionsmodell auf die Bundestagswabl 2009

\begin{tabular}{|l|l|l|}
\hline Partei & Mehrmandate & Mandatsverluste \\
\hline CDU & +1 in BW** & $\begin{array}{l}-1 \text { in } \mathrm{SH}^{*} \\
-1 \text { in SL* }\end{array}$ \\
\hline CSU & +1 in BY** & \\
\hline SPD & +1 in BY & $\begin{array}{l}-1 \mathrm{HH} \\
-1 \mathrm{HB} *\end{array}$ \\
\hline Grüne & $\begin{array}{l}+1 \text { in BE } \\
+1 \text { in BY }\end{array}$ & $-1 \mathrm{HE}$ \\
\hline FDP & +1 in BY & -1 in NI \\
\hline Linke & & . \\
\hline
\end{tabular}

* Durch diesen Verlust entsteht jedoch ein zusätzliches Überhangmandat

** Dadurch Wegfall eines Überhangmandats

Eigene Darstellung

Ohne Einbezug einer im Gesetzentwurf noch vorgesehenen zusätzlichen Reststimmenverwertung wären bei der Bundestagswahl 2009 in Schleswig-Holstein, Hamburg, Niedersachsen, Bremen, Hessen und dem Saarland je ein Proporzmandat weniger vergeben worden als auf Grund des angewandten alten Berechnungsverfahrens. In diesen Ländern waren die Kleinparteien besonders schwach. Nicht so hingegen in Berlin, Baden-Württemberg und Bayern, wo jeweils ein beziehungsweise in Bayern vier zusätzliche angefallen wären. Das heißt im Ergebnis, eine Stimme für die Bayernpartei oder die ÖDP in Bayern nutzt hinterher bei der Proporzverteilung möglicherweise CSU oder SPD. Dieses wäre zwar kein negatives Stimmengewicht, es würde aber zu der skurrilen Situation führen, dass der Wähler einer (regional starken) Kleinpartei dadurch einer anderen Partei zu einem Mandat verhilft. In diesem Sinne würden auch die in einigen Ländern potentiell aussichtslosen Stimmen für die FDP, Grünen und Linken faktisch den eher größeren im selben Land zugutekommen. Der Wähler kann dabei, anders als bei einem Transferable Vote oder einer Nebenstimme, ${ }^{68}$ aber nicht abschätzen, welche Partei davon profitiert. 2009 hätte das die folgenden Effekte nach sich gezogen:

- Die 87.000 Stimmen der NPD in Bayern hätten der CSU ein zusätzliches Mandat zulasten der Linken in Nordrhein-Westfalen beschert (Dadurch wäre ein Überhangmandat bei der CSU weggefallen).

68 Eckhard Jesse, »Das Abschneiden der kleinen Parteien bei der Bundestagswahl 2009 und ihre Perspektiven « in: Oskar Niedermayer (Hg.), Die Parteien nach der Bundestagswabl 2009, Wiesbaden 2011, S. 179-196, hier: S. $193 \mathrm{ff}$. 
- Die 58.000 Stimmen der Piratenpartei in Berlin hätten den Grünen ein zusätzliches Mandat zulasten der Linken in Nordrhein-Westfalen beschert.

- Ohne die 87.000 Stimmen der Piratenpartei in Niedersachsen hätte die FDP in Niedersachsen ein Mandat weniger erhalten und hierfür die Linke ein Mandat mehr in Nordrhein-Westflen.

- Die 33.300 Stimmen der Piratenpartei in Schleswig-Holstein hätten der CDU dort ein Mandat zulasten der CSU beschert. Im Ergebnis wäre ein Überhangmandat von der CDU zur CSU gewechselt.

- Eine Verdoppelung der Stimmen der NPD in Niedersachsen hätte der CDU dort ein weiteres Mandat zulasten eines Mandats der CSU eingebracht, wodurch die CSU aber ein weiteres Überhangmandat erzielt hätte.

Wie absurd und willkürlich die Mandatszuteilung dann vonstatten ginge, zeigen diese Beispiele. Es handelt sich also um einen recht untauglichen Versuch, die machttaktischen Vorteile für das Regierungslager bei der Wahl zu kombinieren mit einer strukturellen Südverschiebung der Proporzmandate.

Unabhängig von der Frage, welche Korrekturen im Zuteilungsverfahren nun abschließend vorgenommen werden, lassen sich daraus jedoch keine grundlegenden Veränderungen im Parteiensystem ableiten, wie es beim Übergang zu einem Grabenwahlrecht oder einem Mehrheitswahlrecht der Fall wäre. Tatsächlich zielt die gegenwärtige Wahlrechtsdebatte einerseits weder darauf ab, das bestehende Parteiensystem zu stabilisieren, noch es besonders zu verändern. Andererseits sorgt die gegenwärtige Struktur des Parteiensystems dafür, dass eine stark systemkonservierende Wirkung im Wahlsystem erkennbar ist. Vor dem Hintergrund der notwendigen Rechtsanpassung läuft die von der Koalition erwogene Lösung gleichwohl darauf hinaus, die Wettbewerbsbedingungen zu ihrem Vorteil zu stabilisieren. Freilich gilt, dass die Parteien und vor allem die Wähler die veränderten Regularien nach einer gewissen Zeit antizipieren. ${ }^{69}$ Stimmensplitting wäre durch eine nach Bundesländern separate Mandatszuteilung jedenfalls hoch attraktiv, weswegen die Zahl der Überhangmandate beträchtlich ansteigen dürfte. Ganz unabhängig von den absurden Effekten der Mandatszuteilung wäre eine strukturelle Festigung des Effekts bei den Überhangmandaten wohl verfassungsrechtlich ebenso hochgradig problematisch wie es vor dem Hintergrund einer drohenden Umkehrung der Mehrheiten geeignet wäre, die Legitimation des politischen Systems zu untergraben. Insofern verändert das Wahlsystem voraussichtlich nicht unmittelbar das Parteiensystem; es wirkt jedoch unterhalb dessen recht nachhaltig auf die Wettbewerbsstrukturen ein.

\section{Fazit}

Zwischen der Wahlsystemdebatte und der Ausformung des Parteiensystems gibt es auffällige Wechselwirkungen. Eine Reform des Wahlrechts geht stets vom gegebenen Parteiensystem aus und die regierenden Parteien sind hierbei bestrebt, für ihre oder ihre

69 Grotz, »Verhältniswahl und Regierbarkeit« aaO. (FN 15), S. 171.

ZfP 58. Jg. 3/2011 
gewünschte Mehrheitskoalition eine möglichst günstige Lösung zu erwirken. Die dadurch benachteiligten Parteien stellen sich am Ende auf die neuen Rahmensetzungen ein und passen daraufhin ihre Strategien an. Die PDS konnte so in einem Wahlsystem überstehen, das eigentlich ihr parlamentarisches Aus zur Folge haben sollte. Nachdem ihre erste Nische durch kleinere Veränderungen verbaut wurde, entstand in der Partei schließlich ein Handlungsdruck, um eine zweite Lücke zu nutzen.

Das Wahlsystem ist folglich darauf ausgelegt, das Parteiensystem hinsichtlich Wettbewerbsbeziehungen und Akteurskonstellationen zu beeinflussen. Nachdem trotz des Wahlsystems 2005 eine Kartellierung des altbundesrepublikanischen Parteiensystems nicht mehr aufrecht zu erhalten war, schuf dann der Richterspruch des Verfassungsgerichts ein Gelegenheitsfenster, um Wahlsystem und Parteiensystem neu aufeinander abzustimmen. Dabei hätte allein in der Großen Koalition noch eine Möglichkeit bestanden, eine umfängliche Wahlrechtsreform zu beschließen, die erheblich zur Strukturierung des Parteiensystems hätte beitragen können. Doch das taktische Verlangen bei der Union war diesbezüglich ebenso stark wie auch die SPD mit Blick auf potentielle Koalitionspartner hieran kein Interesse hatte. Dementsprechend stabilisiert das Parteiensystem einerseits das bestehende Wahlsystem und führt andererseits dazu, dass Reformen im Wahlrecht nur weitgehend konform zum Parteiensystem erfolgen können.

Dennoch beinhalten die verbliebenen Varianten erhebliche Wirkungen auf das Parteiensystem. Das Wahlsystem könnte sich dabei soweit verändern, dass der Parteienwettbewerb sich diesen Gegebenheiten neu anpassen müsste. Die vom Mathematiker Pukelsheim inspirierten Lösungen ${ }^{70}$ würden die aus dem veränderten Parteiensystem aufgetauchte Paradoxie des Wahlsystems beseitigen, wonach die Schwäche der Volksparteien zu einer Zunahme der Überhänge und damit zu einer Überzeichnung von deren Proporz beiträgt. Damit ließe sich eine drohende hochproblematische Umkehrung der Mehrheitsverhältnisse im Bundestag verhindern. Diese Ansätze würden also eindeutig das Wahlsystem an den Strukturen des Parteiensystems neu ausrichten. Das Wahlsystem bliebe im Prinzip erhalten, würde aber die aus dem veränderten Parteiensystem resultierenden Fehler ausgleichen beziehungsweise neutralisieren. Im Ergebnis würde also die These von der Auswirkung des Parteiensystems auf das Wahlsystem bestätigt werden. Der Parteienwettbewerb könnte sich in den bewährten Bahnen abspielen, zugleich wären die aus dem veränderten Parteiensystem resultierenden Funktionsdefekte im Wahlrecht beseitigt.

Demgegenüber würde der Vorschlag, die Mandatszuteilung auf die Länder zu separieren, die Überhangmandate tendenziell fortschreiben. Auch hier würde das Wahlrecht auf das veränderte Parteiensystem reagieren, aber stärker in der Form, dass Anreize gegeben werden für die klare Herausbildung von Lagern und für eine taktische Abgabe der zwei Stimmen. Zwangsläufige Folgen für die Bundestagswahlen muss das alles nicht haben. Auch stellt eine solche Reform keine grundsätzliche Wahlsystemänderung dar, allerdings wird hier das Wahlsystem nicht nur minimalinvasiv geändert. Folgt der Gesetz-

70 Oelbermann / Pukelsheim / Rossi / Ruff , „Eine schonende Verbindung von Personen- und Verhältniswahl zum Abbau negativer Stimmgewichte bei Bundestagswahlen«aaO. (FN 47). 
geber diesem Weg, so ist die Veränderung im Wahlsystem also einerseits Ausfluss aus dem Parteiensystem und wirkt andererseits darauf zurück, indem es Effekte in Bezug auf die Reorganisation der politischen Lager erwarten lässt.

Unterm Strich zeigt sich, dass Parteien- und Wahlsystem sich gegenseitig bedingen und beeinflussen. Es kommt letztlich auf die Umstände und Konstellationen an, was einen Einfluss auf das jeweils andere entfalten kann. Insofern ähnelt die Debatte darum dann doch der berühmten Diskussion um die Henne und das Ei.

\section{Zusammenfassung}

Wahlsystemdebatten prägten die Anfangsjahre der Bundesrepublik, verloren aber in Folge der Konzentration und Stabilität des Parteiensystems an Bedeutung. Dennoch können auch kleinere Änderungen im Wahlrecht einen Effekt auf das Wahlsystem und mittelbar auf das Parteiensystem haben. Die Wechselwirkung zwischen Parteien- und Wahlsystem lässt sich sowohl für die Zeit nach der Bundestagswahl nachzeichnen als auch für die laufende Wahlrechtsnovelle.

Dabei sind eine Reihe von Systemdefekten des Wahlsystems mit der zunehmenden Fragmentierung des Parteiensystems offensichtlich geworden. Bei deren Beseitigung werden die parteipolitischen Interessen deutlich, die jeweils unterschiedliche Lösungsansätze nach sich ziehen.

\section{Summary}

Debates about the voting system left an distinct imprint on the early years of the Federal Republic of Germany, however they lost their relevance as a result of the concentration and stabilization of the party system. Nevertheless, even small changes in the electoral law may have a notable effect on the voting system. This interdependence between the party system and the voting system was apparent for the period after the parliamentary elections (for the Bundestag) as well as for the present process of amendment to the voting laws.

In the course of this development certain defects in the voting system, as a result of the increasing fragmentation of the party system, became clear. In an attempt to adjust these deficits several distinct party political interests are becoming apparent that call for different solutions.

Stephan Klecha, Interrelation between reforms of electoral law and party system 